PROCEEDINGS OF THE

AMERICAN MATHEMATICAL SOCIETY

Volume 134, Number 9, September 2006, Pages 2487-2494

S 0002-9939(06)08426-7

Article electronically published on February 8, 2006

\title{
KAPRANOV RANK VS. TROPICAL RANK
}

\author{
K. H. KIM AND F. W. ROUSH \\ (Communicated by Bernd Ulrich)
}

\begin{abstract}
We show that determining Kapranov rank of tropical matrices is not only NP-hard over any infinite field, but if solving Diophantine equations over the rational numbers is undecidable, then determining Kapranov rank over the rational numbers is also undecidable. We prove that Kapranov rank of tropical matrices is not bounded in terms of tropical rank, answering a question of Develin, Santos, and Sturmfels.
\end{abstract}

\section{INTRODUCTION}

The tropical semiring $(R \cup\{\infty\}, \min (a, b), a+b)$ has many applications but is of special interest in relation to algebraic geometry in terms of valuations. Roughly speaking, a tropical matrix can arise as the matrix of valuations of a matrix of polynomials or power series over a field. In the paper [4 three natural notions of rank are introduced.

Definition 1.1. An $n \times n$ tropical matrix $M$ is nonsingular if and only if the minimum value over permutations $\pi$ of

$$
\sum_{i=1}^{n} m_{i \pi(i)}
$$

is realized uniquely.

If a matrix of power series is singular, then the corresponding tropical matrix must be singular in this sense.

Definition 1.2. The tropical rank of a tropical matrix $M$ is the largest $k$ such that $M$ has a nonsingular $k \times k$ submatrix.

The relationship of the tropical semiring to algebraic geometry starts with a field of power series $K$, where the power series are allowed to have general real exponents in $t$ and the coefficients lie in a base field $F$. The degree map gives a valuation of this ring. Let $\mathcal{I}$ be an ideal in $K\left[x_{1}, \ldots, x_{d}\right]$, corresponding to an algebraic variety. This gives rise to a variety $V(\mathcal{I})$ in $K^{d}$ consisting of (nonzero) $d$-tuples which when substituted for the $x_{i}$ make all elements of the ideal $\mathcal{I}$ equal to 0 . That gives rise to a tropical variety $T(\mathcal{I})$ in Euclidean $d$-space by taking its image under the degree mapping.

Received by the editors March 15, 2005.

2000 Mathematics Subject Classification. Primary 15A99, 16Y60.

Key words and phrases. Kapranov rank, tropical rank. 
For purposes of defining rank the following definition is satisfactory, however [1] gives a more general concept using Grassmannians and hyperplane intersections.

Definition 1.3. A tropical linear space is a tropical variety $T(\mathcal{I})$, where $\mathcal{I}$ is generated by linear forms in the $x_{i}$. Its dimension is $d$ minus the number of minimal generators of $\mathcal{I}$.

Definition 1.4. The Kapranov rank of a tropical matrix $M$ is the least dimension of a tropical linear space containing the rows of $M$.

The Kapranov rank may differ from the tropical rank, but the tropical rank is a lower bound for it. The Kapranov rank is closer to the concept one would like to use in algebraic geometry but does not have such a simple tropical nature. Moreover it can vary according to the field.

Definition 1.5. The Barvinok rank of an $n \times n$ tropical matrix $M$ is the least $k$ such that $M$ is the product of an $n \times k$ tropical matrix and a $k \times n$ tropical matrix.

The Barvinok rank is an upper bound for the Kapranov rank. It is the most natural concept of rank for Boolean matrices, where it also has names such as Boolean rank and Schein rank 7]. The 2-element Boolean algebra is the subsemiring of the tropical semiring consisting of zero and infinity. However if we look at any 2 elements of the tropical semiring, the additive semigroup is isomorphic to the 2-element Boolean algebra, and some properties of matrices whose entries lie in this subset will be like those of Boolean matrices. For basic properties of Boolean matrices, see 7 .

Determining Kapranov rank over a given field involves dealing with nonlinear systems of polynomial equations over that field. Here we will largely deal with the case of Kapranov rank of $(0,1)$-matrices. Develin, Santos, and Sturmfels proved the following.

Theorem 1.6 (4]). The Kapranov rank of $M$ is at most $r$ if and only if $M$ lies in the variety $T\left(\mathcal{J}_{\nabla}\right)$, where $\mathcal{J}_{\nabla}$ is the algebraic variety of matrices of the same size as $M$ over $K$ of rank $r$, and $T$ denotes tropicalization.

That means that there must be some matrix of rank $r$ over the field whose image is $M$. They deduce the corollary that the Kapranov rank is the smallest rank of any lifting over $K$.

\section{KAPRANOV RANK OF MATRICES RELATED to PROJECTIVE Planes}

Proposition 2.1. The Kapranov rank over an infinite field $F$ of an $n \times n(0,1)$ matrix $M$ is the least $k$ such that there exist two families of $k$-dimensional vectors $v\langle s\rangle, w\langle s\rangle$ over $F$ such that $m_{i j}=0$ if and only if the inner product $v\langle i\rangle w\langle j\rangle^{T}=0$, where $T$ denotes transpose.

More generally the Kapranov rank over a field $F$ of an $n \times n$ nonnegative matrix $M$ is the least $k$ such that there exist two families of vectors $v\langle s\rangle, w\langle s\rangle$ over the ring $K$ of power series over $F$ involving arbitrary nonnegative powers of an indeterminate $t$, such that $m_{i j}$ is precisely the order in $t$ of the inner product $v\langle i\rangle w\langle j\rangle^{T}=0$, and here $T$ denotes transpose.

Proof. All entries of $M$ lie in the ring $K_{+}$of power series with no negative degrees. This is a valuation ring in a valuated field, and is a principal ideal domain. Over this ring, modules of $K$-dimension $k$ have also $K_{+}$dimension $k$, by the theory of 
torsion-free finitely generated modules over a principal ideal domain. Therefore a matrix representing $M$ will factor as a product of $n \times k$ and $k \times n$ matrices, $L$ and $R$ over $K_{+}$, one of which gives the module basis for the row space of $M$ and the other gives the linear combinations of that basis which produce all row vectors of $M$. Then the leading terms of $L$ and $R$ will give the required vectors if $M$ does have Kapranov rank at most $k$. In the second case, the converse is immediate.

Conversely (in the former case) suppose that the family of vectors exists. If we add generic degree 1 terms to $L$ and $R$, then the product will have degree 1 wherever the degree is not zero, and we will have the required pattern.

This result extends to the case of any two-valued tropical matrix. It would be of some interest to know whether if $M$ is nonnegative integer-valued, then one can always work with power series having nonnegative integer exponents. If so, then high degree terms could be deleted so that one could also work with polynomials.

Instead of working with two families of vectors, we will equivalently, work with a collection of vectors and hyperplanes through the origin, with the condition that a vector lies in the hyperplane, if we choose the hyperplanes orthogonal to the respective vectors in one of the two sets. That represents some concept of incidence which is natural for projective planes.

The following result is also essentially in [4.

Proposition 2.2. Suppose we have a geometry consisting of a finite collection of points and lines and their incidence (membership) matrix, and suppose there is at most one line through any two points and any two lines that intersect in at most one point. The tropical rank of the incidence matrix of the geometry, taken as a tropical $(0,1)$-matrix, is at most 3 . This remains true if we replace the 1 entries by arbitrary positive real numbers.

Proof. Consider the nature of $4 \times 4$ submatrices. By the Hall-Koenig theorem [6] on systems of distinct representatives, there is at least one system of distinct representatives (matching) for the corresponding binary relation, which consists entirely of zero unless there are configurations of incidence which include $1 \times 4,2 \times$ $3,3 \times 2,4 \times 1$ permuted rectangles where all points are on all lines. Of these only $1 \times 4$ and $4 \times 1$ will occur, and we defer consideration of this case.

So assume the minimum permuted diagonal sum is zero. From [7] on matrices of permanent 1 (the complementary result for Boolean matrices), it follows that if any nonnegative matrix has a unique permuted diagonal sum of zero, then one can permute the rows and columns so that the zeroes lie entirely on or below the main diagonal, and the main diagonal is zero. (This is proved by putting the permuted diagonal on the main diagonal and noting that the graph of the remaining zeroes must be acyclic, hence orderable). But such a pattern means that 2 columns have 2 positive entries in common, two points lie on two different lines.

Now consider the case when the top row consists entirely of positive entries, but no column consists entirely of positive entries. By the condition of the theorem, all the other rows have at most one positive entry. We claim that the minimum permuted diagonal sum equals the minimum entry in the top row, and that it is not unique. Since any sum involves a term from the top row, this is a lower bound on the minimum. Now delete its row and column; we have a $3 \times 3$ matrix with at most one positive entry per row, and no column consisting entirely of positive entries. There cannot be any blocking matrix, so this has a minimum permuted diagonal 
of zero. Moreover it cannot be permuted so that the zeroes are all on or below the diagonal, so it has at least two permuted diagonals of zero, and is singular.

Finally consider the case where the top row and the first column are entirely positive. Then there can be no other positive entries in the matrix. The minimum permuted diagonal sum is either equal to the $(1,1)$ entry or the minimum sum of another entry in the first row and another entry in the first column. In either case, by deleting the rows and columns of those entries, one finds there will be at least two ways to complete the permuted diagonal with zeroes.

For modules over the ring of power series, it will not only be true that a submodule of a finitely generated free module will be free, but that if we have any spanning set for the submodule, some subset of that spanning set will be a basis (spanning and independent over the quotient field). One way to see this is to look at the first coordinate, take a spanning set element of minimum order there, use it as a first basis element, and use it to clear out that coordinate entirely. The resulting module is the kernel of a nontrivial mapping, the projection to that coordinate, and has lower rank over the quotient field. One can repeat this process. This is useful in analyzing certain configurations.

Lemma 2.3. Let $V$ be the algebraic set (subset of an algebraic variety) of all inner products from 2 sequences of $n$ vectors in c-space. Its tropical dimension does not exceed its ordinary dimension which is at most 2 nc.

Proof. This follows from the Bieri-Groves theorem [1, alternatively from the more precise results about tropical Grassmannians in 10]. Note that this set lies in the algebraic variety of $n \times n$ matrices of rank at most $c$; their row and column spaces represent $c$ planes in $n$-space; the entire set is an image of a product of two sets of dimension $c n$.

Theorem 2.4. Over any infinite field there are tropical matrices of tropical rank 3 and arbitrarily high Kapranov rank. These are obtained by taking the incidence matrices of finite projective planes and inserting general positive real numbers for the 1 entries (incidences of points on lines).

Proof. By Proposition 2.2 these matrices have tropical rank 3. The proof that they have arbitrarily large Kapranov rank is essentially done by counting dimensions. Such a matrix $M$ of this type can have arbitrarily large size $\left(m^{2}+m+1\right) \times$ $\left(m^{2}+m+1\right)$ and $\left(m^{2}+m+1\right)(m+1)$ arbitrary positive entries, since each line has $m+1$ points on it. Here $m$ can be any order of a finite field, a power of a prime. If the Kapranov rank is at most $c$, then by Proposition 2.1 there are two families $P, L$, called points and lines, each consisting of $n=m^{2}+m+1$ nonzero vectors in $c$-dimensional space over a power series ring $R$ over the given infinite field, in a variable $t$, such that the orders in $t$ of the inner products of the points with the lines are given by the matrix $M$. The set of all possible inner products has ordinary dimension at most $2 n c$ by choice of each of $c$ coordinates for each of $n$ points and lines, as in Lemma 2.3, but the dimension of the set of all matrices of this type is equal to the number of incidences in the projective plane, asymptotically $n^{3 / 2}$. This gives a contradiction. 


\section{Diophantine Equations}

Hypothesis. Diophantine equations over the rational numbers are not decidable by a general algorithm.

This problem is unknown and very deep. Davis, Putnam, and Robinsion, and Matijasevitch proved (Hilbert's Tenth Problem) that Diophantine equations over the integers are algorithmically undecidable. To relate this to Kapranov rank, we note by the above that representing any system of incidence of lines and points in a projective plane is a problem of deciding if Kapranov rank is at most 3. Then we make use of Hall's method given in 5 for coordinating projective planes and defining algebraic operations solely in terms of systems of intersections of points and lines.

The method of Hall to assign coordinates to a projective plane starts by choosing 4 points $X, Y, O, I$, no three collinear, where $O$ is assigned coordinates $(0,0), I$ is given coordinates $(1,1), O X$ and $O Y$ are the $x$ and $y$-axes, and $X Y$ is understood as the line at infinity. The points on $O I$ other than its intersection denoted (1) with $X Y$ are given coordinates $(b, b)$. All points $P$ of the plane not on $X Y$ are given their $x$ and $y$ coordinates by intersecting $X P$ (a horizontal line) and $Y P$ (a vertical line) with $O I$. Addition is defined by setting $y=x+b$ if and only if $(x, y)$ is a finite point on the line joining (1) with $(0, b)$. Multiplication is defined by setting $y=x m$ if and only if $(x, y)$ is a finite point on the line joining $O$ and the infinite point $(m)$ which is the intersection of the line joining $(0,0)$ and $(1, m)$, with $X Y$. These operations agree with standard operations of arithmetic for the standard projective plane over any field. We can take a projective transformation so that any given finite set of points and lines are finite in the sense of not lying on $X Y$.

For further information on Diophantine undecidability over the rational numbers, see 8 .

Theorem 3.1. Determining whether a two-valued tropical matrix has Kapranov rank 3 over an infinite field has precisely the computational complexity of solving a system of Diophantine equations over that field. Under the above hypothesis, computing Kapranov rank over the rational numbers is algorithmically undecidable. Computing Kapranov rank of an $n \times n(0,1)$-matrix over any infinite field is $N P$ hard.

Proof. For a $(0,1)$-matrix, having Kapranov rank 3 over the rational numbers is equivalent by Proposition 2.1 to finding two systems of 3-dimensional vectors over the field in question whose zero and nonzero inner products represent it, and as remarked above, to finding a system of nonzero vectors and planes through the origin in 3 space whose incidence relations represent it. For a finite system, we can move all the vectors and planes away from infinity, and represent them in terms of a system of points and lines in the plane (or projective plane) over that field.

We use Hall's construction to represent a general Diophantine equation with positive integer coefficients on each side as a question of existence of a system of points and lines with suitable intersections and nonintersections. We choose 4 points as above to define the system, with the non-collinearity assumption. Then we choose a number of general points sufficient to define the number of variables required. Then we take the lines and intersections required to define each side of 
the equations as a point on $O I$. Existence of this configuration is then equivalent to solving the Diophantine system. Some incidences and intersections have been undefined, but if we take all possibilities for 0 and 1 on the incidence matrix for them, it cannot be possible to algorithmically decide all the cases.

The same argument proves NP-hardness in general, given that solving Diophantine equations or systems over a field of polynomial size, with coefficients and exponents having polynomially many digits, is NP-hard. This follows by taking equations representing a Boolean satisfiability problem, and adding equations $x^{2}=x$ to force the solutions to be 0 or 1 .

However, in the NP case it is not sufficient to allow all possibilities for the unknown incidences, since there might be exponentially many. Instead, we can control them by adding new variables and equations, and then taking linear combinations of the given equations. Our addition and multiplication constructions define certain points and lines and incidences of these. In particular cases there may be additional incidences of a point and a line. But if so, this will be because of the separate constructions involved in one or two equations, not the entire system if there are many equations. Transform the equations as in 9 to be linear-quadratic, and arrange that the nth variable, instead of being 0,1 , has $2^{n}+1$ added to it so that it is either $2^{n+2}+1$ or $2^{n+2}+2$.

Assume first that the field has transcendentals, indeterminate or generic elements. Choose all equations that consist of one side set equal to zero. Add 2 new variables, and 2 new equations $E_{1}, E_{2}$ in them, which in effect define the new variables as generic and independent quadratic combinations of the other variables. Replace the previous equations by their sums with generic linear combinations of the new equations $E_{i}$; the combined equations have the same quadratic form as the $E_{i}$. Do the addition and multiplication constructions so as to compute in some fixed order all the new coefficients in each equation and their products with products of at most 2 previous variables and then add them successively, and finally add in the linear terms in the 2 new variables. The coordinates of points and coefficients of line equations will represent partial steps in the computation, or separate variables or certain fixed constants or transcendentals for the coefficients. It will not matter for our proof exactly what the incidences in each equation or pair of equations are, just that they can be computed in polynomial time and that they will not depend on which variables have one of the two values versus which variables have the other.

Note that we do not have to construct the numerical values of the variablestheir existence alone is what is needed to see whether this configuration is possible and to see whether the Diophantine equations are solvable. We also do not have to construct the transcendentals, their existence is enough. Also, the linear combinations which we take are nonzero so that we can recover solutions of the original equations from solutions of the new equations. However we must construct the coefficients as polynomial combinations of the transcendentals in them, and all integers in them starting with 1 . In the next two paragraphs we argue that for 2 different equations, or at two different terms for same equation, the partial steps in the computation will involve different transcendentals, and we can have no new incidence among them. For different partial computations of the same term in the same equation, there are only the natural incidences, not ones depending on values of the variables. The incidences involving variables will be only that the same variable has the same value in two different equations. 
The addition construction can be arranged to construct in turn, using some parallelism, from $(a, a),(b, b), x=a,(a, 0), x=y+a, y=b,(a+b, b), x=a+$ $b,(a+b, a+b)$. Thus it adds the following varying finite points in order to add $a, b$ : $(a, a),(b, b),(a, 0),(a+b, b)$, and the following finite lines: $x=a, x=y+a, y=$ $b, x=a+b$. The multiplication construction can be arranged to construct in turn $x=1, y=a,(1, a), y=a x, x=b,(b, a b), y=a b,(a b, a b)$. Thus it adds the following finite points in order to multiply $a, b:(a, a),(b, b),(0,0),(1,1),(1,0),(1, a),(b, a b)$, $(a b, a b)$ and these lines: $x=1, y=a, y=a x, x=b, y=a b$. We can choose in these that $a$ is a partial computation carried from before, and $b$ is a coefficient or variable, or the integers $-1,1$ or 2 . We do computations of terms in order, with the new variables in $E_{i}$ last; when each term is complete, add it to the previous partial sum. Consider a term as some $\left(\sum_{r, s} n_{r s} t_{r} t_{s}\right) x_{i} x_{j}$, where $t_{r}, t_{s}$ are transcendentals (there may be only one), $x_{i}, x_{j}$ are variables (there may be one or none), and $n_{r s}$ are integers. Lowest powers of 2 are added first in computing binary integers. For subtraction, we multiply a coefficient, after computing it, by a fixed -1 (a fixed diagram verifies its sum with 1 is 0 ). First the transcendentals $t_{i}$ are multiplied, and among those, first transcendentals from the $E_{i}$, then the multiples by them of the powers of 2 in the binary expansions of $n_{r s}$, and then those are added. Once the entire polynomial coefficient is computed, it is multiplied times $x_{i}, x_{j}$, over which we have little control beyond knowing their two possible values.

The lines above are either vertical or horizontal or at a 45 degree angle with intercepts $0,1, a, b, a+b, a b$, or have the form $y=a x$. The points lie on pairs of these lines, and their coordinates are all $0,1, a, b, a+b, a b$. This means for incidences (in addition to partial computations being equal, we need only to look at ratios $(a+b) / b$ when a sum is being computed), for incidences on some $y=a_{1} x$ and differences $1-a, b-a b$ when a product is being computed, for incidences on some $x=y+a$; the second coordinate is always the more advanced in a product and the less advanced in a sum, in the partial computation. Every line or point involving an $a$ involves a new transcendental or linear combination of transcendentals in a new term: these partial computations will not be the same as those for another equation or another term in the same equation, nor can they equal a variable. This is also true for $a-1, a b-b$. As things are arranged, new transcendentals will not cancel from $(a+b) / b$ and will not give the value of any variable; all the more, this cannot happen when we are adding a previous collection of terms. Such $a+b$ cannot occur in the stages of multiplying in the variables, and cancellation will not occur in adding in a completed term. In the process of multiplying out a particular term within itself there cannot be any unpredictable incidences involving the variables, since multiplying them is done last. This shows that all incidences are computable in polynomial time from a knowledge of the equations, without knowing the values of the variables.

Solvability of the new system means precisely realizability in terms of a system of points and lines over the field, hence Kapranov rank at most 3 of the corresponding $(0,1)$-matrix. Hence if there were a $\mathrm{P}$ algorithm for Kapranov rank 3, then there would be a $\mathrm{P}$ algorithm for solving a system of Boolean or Diophantine equations in terms of 0,1 which is an NP-complete problem.

If we have a nonalgebraically closed but infinite field, then a polynomial number of trials of substitutions of field elements for transcendentals one by one 
will result in some case where each of the polynomially many intersections remain distinct.

This extends to the case in which the matrix consists of integers of at most polynomial size. For larger sizes we must solve equations in terms of power series which are zero over large ranges, and the result is less clear. If it is a question of extending linearly after a very few stages, then it can be done.

It has previously been proved that determining Barvinok rank 3] and tropical rank 9] are in general NP-hard problems. It would also be interesting to know what the general form of a nonnegative matrix of small tropical rank is, when it is not given directly by the pattern of zeroes.

It would also be interesting to know whether the Kapranov ranks over the complex numbers of a sequence of projective planes over finite fields represented as tropical $(0,1)$-matrices tends to infinity. If so, then there can be no purely tropical definition of any lower bound $r_{0}$ on Kapranov rank over every field such that given $r_{0}$ there is an upper bound $U\left(r_{0}\right)$ on Kapranov rank, for the Kapranov ranks over fields of different characteristic will not be mutually bounded, but the tropical matrices of projective planes in this sense are field-independent. However it appears that there will be a tropically computable lower bound which can deal with the dimensionality phenomenon of Theorem 2.4, and in that sense improves on Kapranov rank.

This is related to the topic of realizable matroids. J. Canny 2 has some results on the computational complexity of solving Diophantine systems over the real and complex numbers.

\section{REFERENCES}

[1] R. Bieri and J.R.J. Groves, The geometry of the set of characters induced by valuations, J. reine und angewandte Mathematik 347(1984),168-195. MR0733052 (86c:14001)

[2] J. Canny, Some algebraic and geometric computations in PSPACE, Proc. 20th Symp. Theory of Computing, Chicago(1988),ACM Press.

[3] E. Çela, R. Rudolf, and J. Woeginger, On the Barvinok rank of matrices, presentation at the 2nd Aussois Workshop on Combinatorial Optimization, February 1998.

[4] M. Develin, F. Santos, and B. Sturmfels, On the rank of a tropical matrix, arxiv:math.CO/0312114.

[5] Marshall Hall, Group Theory, Macmillan, New York, 1964.

[6] Philip Hall, On the representatives of subsets, J. London Math. Soc. 10(1935),26-30.

[7] K. H. Kim, Boolean Matrix Theory and Applications, Marcel Dekker, New York, 1982. MR0655414 (84a:15001)

[8] K. H. Kim and F. W. Roush, Problems equivalent to rational Diophantine solvability, Proc. A.M.S 124, no. 2 (1989),493-505. MR.1011609 (90i:03048)

[9] K. H. Kim and F. W. Roush, Factorization of polynomials in one variable over the tropical semiring, arxiv:math.CO/0501167

[10] D. Speyer and B. Sturmfels, The tropical Grassmannian, math.AG/0304218.

[11] D. Speyer and B. Sturmfels, Tropical mathematics, arxiv:math.CO/0408099.

Department of Mathematics, Alabama State University, Montgomery, Alabama 36101-0271 - And - Fellow, Korean ACAdemy of Science and Technology

E-mail address: khkim@alasu.edu

Department of Mathematics, Alabama State University, Montgomery, Alabama 36101-0271

E-mail address: froush@alasu.edu 